\title{
Unternehmen zwischen Resignation und Hoffnung - Ergebnisse einer Fallstudie in einem Moskauer Betrieb*
}

\author{
Sabine Boerner / Diether Gebert ${ }^{* *}$
}

Die gegenwärtige Phase des russischen Transformationsprozesses wird als ein Übergangsstadium zwischen der geschlossenen und der offenen Gesellschaft interpretiert. Anhand von Interviews mit Beschäftigten in einem Moskauer Unternehmen wird dokumentiert, wie die Betroffenen diesen kritischen Zustand subjektiv erleben und welche Handlungsoptionen sie angesichts dieser Lage wählen. Diese Handlungsmuster werden unter Bezug auf das Modell von Lazarus (1991) als Bewältigungsversuche von Bedrohung interpretiert. Es zeigt sich, daß die vorherrschenden Reaktionsweisen wenig dazu geeignet sind, zu einer Entschärfung der problematischen Lage beizutragen. Vor diesem Hintergrund werden Anhaltspunkte für die Führung von Mitarbeitern im Stadium des "Dazwischen" erörtert.

The current phase of the Russian transformation process is interpreted as a transition stage between the closed and and the open society. Having interviewed people employed in a Moscow company, the authors document how those concerned are subjectively experiencing these critical circumstances and which alternatives for action they choose. Drawing on a model by Lazarus (1991), the authors interpret the patterns of action as attempts to cope with threat. The prevailing responses prove to be poorly suited to helping defuse the problematic situation. Aspects of leadership during transitions are discussed on this basis.

* $\quad$ Manuscript received: 24.2.97, revised: 1.7.97, accepted: 14.7.97

** Sabine Boerner, geb. 1963, Dr. rer. oec., Technische Universität Berlin, Institut für Betriebswirtschaftslehre, Fachgebiet Organisation, Personalwesen und Führungslehre. Forschungs- und Arbeitsschwerpunkte: Organisationskultur, Organisationales Lernen, Organisationaler Wandel.

Adresse:TU Berlin, Fachbereich 14 - Wirtschaft und Management, Fachgebiet Organisation, Personalwesen und Führungslehre, Straße des 17. Juni, 10623 Berlin Email: boerner@perform.ww.TU-Berlin.DE

Diether Gebert, geb. 1940, Prof. Dr. phil., Technische Universität Berlin, Institut für Betriebswirtschaftslehre, Fachgebiet Organisation, Personalwesen und Führungslehre. Forschungs- und Arbeitsschwerpunkte: Führungsforschung, sozialer Wandel, Organisationsentwicklung. 
Der Transformationsprozeß in der russischen Föderation ist seit einiger Zeit Gegenstand auch der westlichen Managementliteratur (z. B. Hentze/ Lindert 1992; Tatur 1992; Götz 1991). Dabei wird die gegenwärtige Situation der russischen Wirtschaft und der einzelnen Unternehmen häufig als eine Station auf dem Weg in Richtung "Westen" interpretiert, womit sich zahllose Bemühungen legitimieren, Führungskräften Ratschläge aus westlicher Sicht zu erteilen und diese in entsprechenden Managementkursen sowohl vor Ort als auch im Westen einzuüben (Bogacheva 1993). Derartige Bestrebungen unterliegen mitunter der Gefahr, daß zugunsten des reinen Transfers von Managementwissen die speziell russischen Kontextfaktoren $\mathrm{zu}$ wenig Berücksichtigung finden (Peng/ Heath 1996; Pieper 1993; Welsh et al. 1993).

Der nachstehende Beitrag versteht sich insofern als Ergänzung, als hier die Diagnose des Status quo im Vordergrund steht. Dabei geht es jedoch nicht um eine Beschreibung der situativen Faktoren aus objektiver Sicht, sondern es soll untersucht werden, wie russische Führungskräfte und Mitarbeiter die heutige Situation subjektiv wahrnehmen und verarbeiten. Damit zielt unser Beitrag primär auf die Dokumentation eines Bewußtseinszustandes. Die Erhebung dieses Bewußtseinszustandes ist wichtig, weil dadurch bestimmte Handlungsmuster innerhalb der Transformation erklärlich werden, die ihrerseits auf den weiteren Verlauf der Transformation Einfluß nehmen können.

Auf der Basis (halbstandardisierter) Interviews in einem Moskauer Bergbauunternehmen wird eine Positionsbestimmung versucht, die sich auf zwei Fragenkreise konzentriert.

Im Vordergrund steht erstens die Frage, wie die betroffenen Führungskräfte und Mitarbeiter die gegenwärtige Phase der Transformation subjektiv erleben und bewerten. Es zeigt sich, daß in der Wahrnehmung der Betroffenen keinesfalls (euphorische) Aufbruchstimmung vorherrscht, sondern im wesentlichen die Erfahrung von Verlust und Enttäuschung sowie das Gefühl einer Blockierung von Handlungsmöglichkeiten dominieren. Vor diesem Hintergrund werden zweitens die in der heutigen Situation vorherrschenden Handlungsmuster der Akteure erfragt und als für die gegenwärtige Situation typische Bewältigungsversuche von Bedrohung interpretiert.

Unsere Ergebnisse zeigen, in welchem Sinne die Befragten die Lage als schwierig erleben und daß viele der berichteten Handlungsstrategien die Schwierigkeiten eher vergrößern als verkleinern dürften.

\section{Ausgangsüberlegungen und Bezugsrahmen}

Obwohl in diesem Beitrag nicht die Überprüfung oder Weiterentwicklung einer Theorie im Vordergrund steht, sei der theoretische Bezugsrahmen, innerhalb dessen wir unsere Ergebnisse abbilden, kurz skizziert. 
Abbildung 1: Grundannahmen der offenen und der geschlossenen Gesellschaft und ihre Konkretisierung

geschlossene

Gesellschaft offene

Gesellschaft

A. Die anthropologische Dimension

\begin{tabular}{|l|}
\hline Stabilität, \\
Voraus- \\
schaubarkeit
\end{tabular}

\begin{tabular}{rl|l|} 
deterministisch & $\Leftrightarrow \quad$ voluntaristisch & $\begin{array}{l}\text { Prinzip } \\
\text { Hoffnung, } \\
\text { Innovation }\end{array}$ \\
\hline
\end{tabular}

\section{B. Die soziale Dimension}

II.Vorherrschend ist

Harmonie
Interessenhomo $\Leftrightarrow$ genität

$$
\begin{gathered}
\text { Interessen- } \\
\text { heterogenität }
\end{gathered}
$$
Pluralität

II. Die Menschen sind \begin{tabular}{|l|l|l|}
\hline $\begin{array}{l}\text { stabiles Rollen- } \\
\text { gefüge }\end{array}$ & ungleichwertig $\Leftrightarrow \quad$ gleichwertig & $\begin{array}{l}\text { Chancengleich- } \\
\text { heit }\end{array}$ \\
\hline
\end{tabular}

IV. Schutzbedürftig ist Sicherheit, Ordnung

das Kollektiv $\Leftrightarrow \quad$ der einzelne $\begin{aligned} & \text { Individualität, } \\ & \text { Freiheit }\end{aligned}$

\section{Die erkenntnistheoretische Dimension}

Eindeutigkeit, Sinn

Die politischen und wirtschaftlichen Umbrüche, die sich im Rahmen des russischen Transformationsprozesses vollziehen, können als eine Bewegung von einer geschlossenen in Richtung auf eine offene Gesellschaft interpretiert werden (Gebert/ Boerner 1995). Die alte Sowjetunion entsprach in vielen Punkten dem Muster einer geschlossenen Gesellschaft (vgl. Abb. 1). So 
bedeuteten die Zentralverwaltungswirtschaft und die Vorherrschaft der kommunistischen Partei, daß Menschen sich nicht als Subjekt, sondern vor allem als Objekt staatlicher Gewalt und Bevormundung erlebten (Shubkin 1992); auf der sozialen Dimension wurde der Schutz des Kollektivs vor die Förderung des Individuums gestellt (Vlachoutsicos/ Lawrence 1996). Schließlich wurde mit der ideologischen Vorherrschaft von Staat und Partei die Überzeugung vermittelt, es gebe unantastbare Gewißheiten und letztgültige Wahrheiten. Im Rahmen der Transformation wurden einige Merkmale dieser geschlossenen Gesellschaft (z. B. Zentralverwaltungswirtschaft, Vorherrschaft der Partei) in Richtung auf eine offene Gesellschaft verändert (Bayer 1991), deren Idealform sich mit folgenden Stichworten umreißen läßt: Voluntarismus (Mensch als Subjekt), Individualismus und Vorläufigkeit menschlicher Erkenntnis, Lernen und Toleranz gegenüber abweichenden Meinungen. Dennoch kann unter den gegenwärtigen Bedingungen und gemessen an den bisherigen Transformationserfolgen die russische Gesellschaft nicht als eine offene Gesellschaft (etwa im Sinne westlicher, demokratischer und marktwirtschaftlicher Gesellschaften; vgl. Hensel 1992; Cassel 1988) bezeichnet werden. Vielmehr scheint die gegenwärtige Lage die Position des "Dazwischen" zu illustrieren.

Vor diesem Hintergrund lautet unsere Überlegung wie folgt: Interpretiert man die zitierten Gesellschaftstypologien aus der Sicht des Individuums jeweils als Beschreibung der Möglichkeiten und Grenzen der Verfolgung eigener Lebensinteressen, so wird offensichtlich, daß bereits das Leben in einer der beiden Ordnungen (der offenen oder der geschlossenen Gesellschaft) als zwiespältig und widersprüchlich erlebt werden kann, weil jede der beiden Ordnungen gleichzeitig Vor- und Nachteile aufweist. Da beide Muster attraktiv sind, findet man in der Realität Mischungen und Kombinationen dieser beiden Muster und nicht deren idealtypische Realisierung vor. Allein schon der Mischungscharakter erleichtert das Leben auch in der überwiegend geschlossenen Struktur trotz prinzipiell verbleibender Grenzen der Verfolgung eigener Lebensinteressen. Im übrigen ist mit Nachdruck zu betonen, daß das Muster der geschlossenen Gesellschaft in seinen positiven Ausprägungen zunächst einmal von vielen als in hohem Maße attraktiv erlebt wird, weil es z. B. Orientierung, Sicherheit und das Gefühl der Zugehörigkeit vermittelt (Gebert/ Boerner 1995). Es kommt hinzu, daß sich die Begrenzungen und Nachteile im Längsschnitt betrachtet erst im Verlaufe der Zeit als Folge der geschlossenen Praxis entwickeln, und schließlich ist zu bedenken, daß im Zuge langjähriger Sozialisationsprozesse Bewertungsraster vermittelt werden, die es selbstverständlich ermöglichen, daß das Leben in einer überwiegend geschlossenen Struktur eine Zeitlang trotz seiner Begrenzungen als per Saldo befriedigend erlebt wird. 
Für das Stadium des "Dazwischen" gelten nun aber Besonderheiten, die das Leben erschweren: Öffnungsprozesse werden u. a. von der Hoffnung auf die Überwindung der Begrenzungen getragen, die für die geschlossenen Strukturen charakteristisch sind. Öffnungsprozesse sind damit aber nicht nur Anreiz; Öffnungsprozesse sind im Sinne unserer Modellannahme dagegen prinzipiell auch insofern bedrohlich, als sie mit dem Verlust der Güter der geschlossenen Strukturen zu bezahlen sind. Der Zustand des "Dazwischen" bedeutet also zum einen, daß bestimmte gewohnte Vorzüge des alten Systems verloren zu gehen drohen, wobei diese Vorzüge in der nostalgishen Rückwärtsschau häufig auch noch glorifizierend überzeichnet werden. Zum zweiten gilt (und dies zeigte sich nach unseren Beobachtungen sehr anschaulich auch im Rahmen der Wende in der Bundesrepublik 1989; vgl. die Interview-Ergebnisse bei Gebert/ Boerner 1995), daß im Rahmen des Öffnungsprozesses die Vorzüge des offenen Modells idealisiert werden, so daß Enttäuschungen vorprogrammiert sind. Der Zustand des "Dazwischen" konfrontiert den einzelnen dabei nicht nur mit der Erfahrung, daß die anvisierten Vorteile des alternativen Modells noch nicht vollständig erreicht sind, der Zeitbedarf für die Realisierung der angestrebten Ziele also unterschätzt wurde; hinzu kommt, daß selbst die bereits eingetretenen Züge des alternativen Modells nicht unbedingt das halten, was sie zu versprechen schienen.

Wir nehmen entsprechend an, daß sich im Zustand des "Dazwischen" in zweifacher Weise eine aversive Situation vermittelt und gehen davon aus, daß die heutige Lage von den Befragten als bedrohlich wahrgenommen wird. Vor diesem Hintergrund soll in unserer Untersuchung geklärt werden, in welcher Hinsicht die Lage als aversiv bzw. als bedrohlich erlebt wird. Im Sinne unseres Ziels, die Bewußtseinsstruktur der Handelnden genauer zu dokumentieren, soll untersucht werden, was aus ihrer Sicht im Zuge der Transformation verlorenging und was ggf. dazugewonnen wurde.

Bezüglich der bedrohlichen Situation stellt sich die Frage, wie die russischen Befragten mit dieser Situation umgehen, wobei wir uns nachstehend an dem Bedrohungsbewältigungsmodell von R. S. Lazarus (1991) orientieren. Es handelt sich um ein psychologisches Modell, in dem, wie in der Abb. 2 illustriert, verschiedene Strategien unterschieden werden, die als Bewältigungsversuch im Umgang mit bedrohlichen Konstellationen in Frage kommen.

Der Transformationsprozeß vermittelt sich im Sinne unserer Modellannahme in der Phase des "Dazwischen" (objektive Situation) in eine partiell aversive Situation, die als potentiell bedrohlich eingestuft wird (1. Bewertung). Bei der zweiten Bewertung schätzt die Person ab, ob sie über genügend Situationskontrolle verfügt, um die für sie bedrohliche Situation zu bewältigen. Werden die eigenen Handlungsmöglichkeiten als nicht ausreichend eingeschätzt, so sind die Handlungswege blockiert und die Person reagiert mit intrapsychischer Anpassung (resignative Anpassung bzw. Beschönigung der 
Realität) mit der möglichen Folge, daß das bisherige Problem für die Person subjektiv nicht mehr existiert. Werden die eigenen Kräfte größer als die von der Problemstruktur abverlangten Kräfte eingeschätzt, wird Initiative in Form von "Angriff" erfolgen; werden die eigenen Kräfte dagegen als zu gering eingeschätzt, um das Problem zu lösen, reagiert die Person mit der Initiative "Flucht". Das Angriffsverhalten führt zu einer Veränderung der bedrohlichen Situation und trägt damit zu einer objektiven und subjektiven Problementschärfung bei. Das Fluchtverhalten dagegen kann zwar aus Sicht des einzelnen problementschärfend wirken, weil sich der einzelne der bedrohlichen Situation entzieht, trägt jedoch insgesamt nichts zur Problementschärfung bei, sondern kann aus Sicht des übergreifenden Subsystems (z. B. des Betriebs oder der Volkswirtschaft) sogar zur Eskalierung des Problems führen. Prozesse der Resignation können subjektiv zu einer deutlichen Problementschärfung, objektiv aber natürlich auch zu einer Problemstabilisierung beitragen.

In dieser Untersuchung interessiert uns, welche der von Lazarus unterschiedenen Bedrohungsbewältigungsstrategien in der Stichprobe der russischen Befragten im Vordergrund stehen und wie sich diese Präferenzen durch die Spezifika der aktuellen Situation in Rußland (als Zustand zwischen der geschlossenen und der offenen Gesellschaft) erklären lassen. Auf dieser Basis soll anschließend gefragt werden, ob und in welcher Hinsicht sich Anhaltspunkte für die Führung von Mitarbeitern in dieser Phase des "Dazwischen" ergeben.

Wir nehmen in unseren Modellannahmen auf einen allgemeinpsychologischen Erklärungsansatz Bezug, weil die subjektive Verarbeitung der objektiven gesellschaftlichen und betrieblichen Situation und die Wahl der jeweiligen Bedrohungsbewältigungsstrategie als ein psychologischer Prozeß zu verstehen ist. Wir beziehen uns dabei speziell auf das Bedrohungsbewältigungsmodell von Lazarus, weil es solche Coping-Strategien in den Vordergrund stellt, die für die weitere Entwicklung des Transformationsprozesses bedeutsam sind. Natürlich soll auf diesem Wege der Transformationsprozeß nicht unangemessen psychologisiert werden, sondern nur durch eine psychologische Perspektive ergänzend beleuchtet werden. Der Zusammenhang zwischen der objektiven gesellschaftlichen Situation und der psychologischen Verarbeitung dieser Lage liegt systematisch darin, daß der Zustand des "Dazwischen" nicht nur eine bestimmte Konstellation aufbaut, die als mehr oder weniger aversiv (potentiell bedrohlich) erlebt wird (1. Bewertung), sondern zugleich eine Konstellation aufbaut, die über ein Mehr oder Weniger an Situationskontrolle entscheidet (2. Bewertung). 
Abbildung 2: Bewertung der Situation und Handlungsoptionen in Anlehnung an Lazarus (1991).

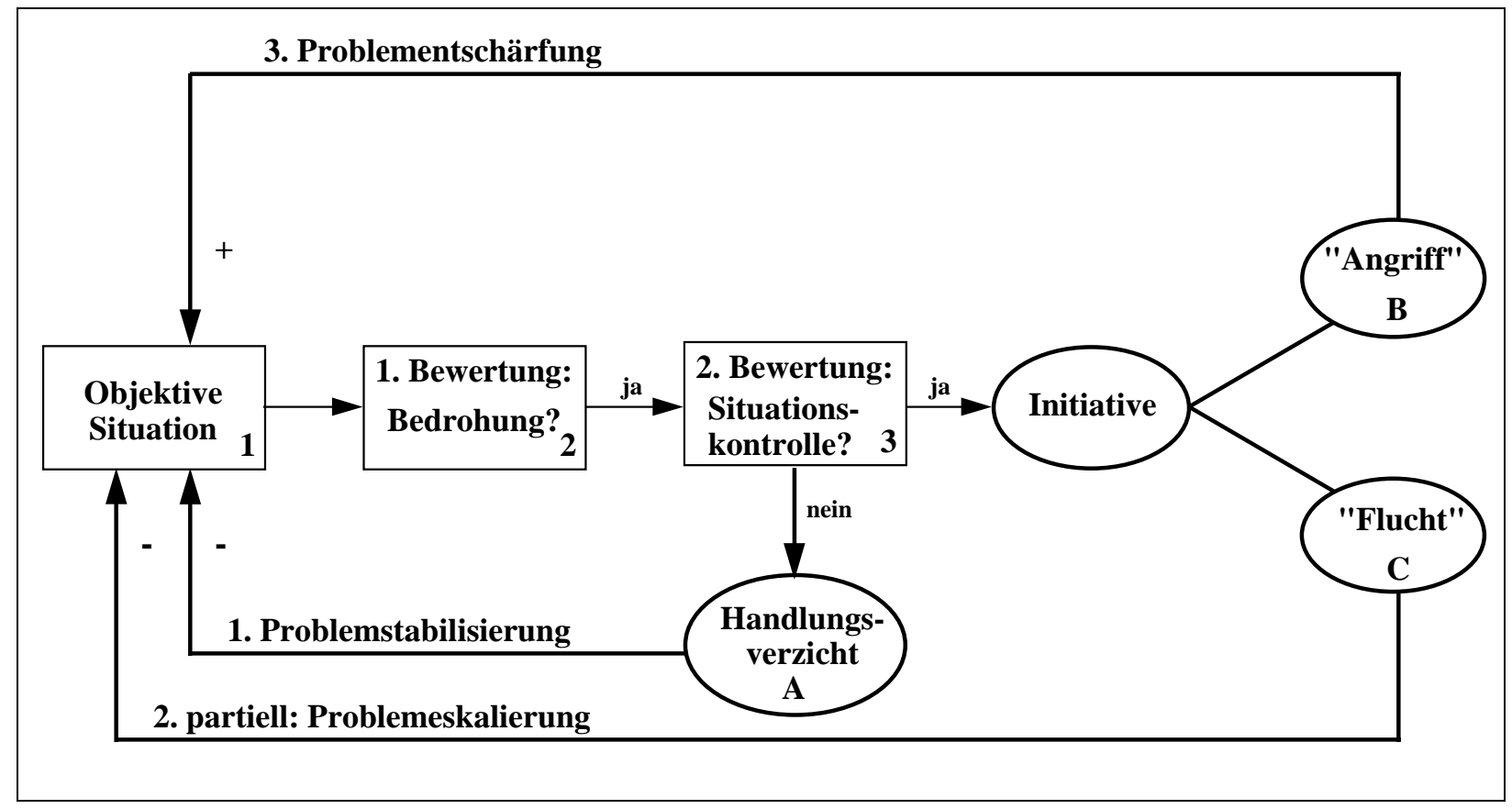

\section{Stichprobe und Art der Befragung}

Die Befragung fand im Herbst 1996 in einem ehemaligen Staatsbetrieb der Bergbaubranche statt, der in der Nähe von Moskau angesiedelt ist. Befragt wurden 15 Personen, von denen einige zum obersten Management gehörten (drei stellvertretende Generaldirektoren), fünf Führungskräfte mit Personalverantwortung (Abteilungsleiter), drei Mitarbeiter mit und vier ohne Personalverantwortung. Die Interviews wurden mit Hilfe einer Dolmetscherin durchgeführt, wobei jeweils die Gespräche zwischen der Dolmetscherin und der Interviewerin dokumentiert wurden (Boerner/Gebert/Agafonov 1996) ${ }^{14}$. Da es sich insofern um einen Dialog über einen Dialog handelt, erscheint der Befragte in den Gesprächen jeweils in der dritten Person.

Mit dem befragten Unternehmen wurde eine eher konservative Branche ausgewählt, die sicherlich nicht als repräsentativ für den aktuellen Zustand der russischen Wirtschaft betrachtet werden kann. Der Vorteil dieses Vorgehens liegt darin, daß gerade in dieser Branche entsprechend unserer theoretischen Annahmen der Zustand des Dazwischen in seiner Qualität besonders prägnant beschreibbar ist, weil in einer konservativen Branche der Sprung in das offene Muster vermutlich als besonders konfliktträchtig erlebt wird.

${ }^{14}$ Wir danken Dr. Jury Agafonov und Olga Nowikowa, die uns bei der Durchführung der Interviews unterstützten, sowie Martina Albrecht, Barbara Behrendt und Birgit König für die Transkription der Interviews. 
Unter der Annahme, daß die Bewertung der Lage und die gewählten Handlungsalternativen nicht nur von den Gegebenheiten in dem Unternehmen selbst abhängen, sondern auch von der Beurteilung des gesamten Lebensumfeldes, haben wir in unseren Fragen die Einschätzung der Gesamtlage im Zuge der wirtschaftlichen und politischen Veränderungen mitberücksichtigt. Im folgenden werden Ausschnitte aus diesen Interviews nicht nur jeweils zur Illustration der Argumentation zitiert, sondern auch, um die vorgefundene Bewußtseinslage ausführlich zu dokumentieren.

\section{Zur Beschreibung des Status quo im untersuchten Betrieb}

Malachowka (gegründet 1945) war als Staatsbetrieb früher Teil eines Verbundes, der außerdem aus dem staatlichen Kohleministerium und einer staatlichen Forschungseinrichtung bestand. Die Aufgabe von Malachowka bestand darin, nach den Plänen dieser Forschungseinrichtung neue Spezialmaschinen für den Bergbau (z. B. Förderbänder, Stempel) zu bauen und diese auf ihre Tauglichkeit unter bestimmten Bedingungen untertage (z. B. bestimmte Druckverhältnisse, unterschiedliche Dicke der Kohleflöze, bestimmte Neigungen der Flöze) zu testen. Mit seinen Testeinrichtungen war Malachowka einmalig in der ehemaligen UdSSR (70\% der Bergbaumaschinen eines bestimmten Typs wurden in Malachowka hergestellt). Es wurden auch Maschinen aus anderen Ostblock-Ländern getestet. Insofern besaß Malachowka als innovatives Unternehmen auf dem russischen Markt und innerhalb des Ostblocks eine Sonderstellung.

Durch den Transformationsprozeß ist der ehemalige Forschungsverbund zusammengebrochen. Formal wurde die starke Abhängigkeit vom Staat reduziert. Malachowka wurde in eine Aktiengesellschaft umgewandelt, wobei der Staat 38\% der Aktien besitzt (25\% wurden kostenlos an die Belegschaft vergeben; 20\% von der Unternehmensführung übernommen; die restlichen 17\% gingen in den freien Verkauf). Wie bei anderen russischen Privatisierungen auch (Götz 1991), besteht de facto jedoch weiter eine starke Abhängigkeit, weil der Staat der hauptsächliche und vor allem im Hinblick auf die Bezahlung der sicherste Abnehmer ist.

Durch den Transformationsprozeß ist auch die traditionelle Struktur aus Beschaffung, Produktion und Absatz weitgehend zusammengebrochen, die früher im Tauschverfahren abgewickelt wurde (z. B. Maschinen gegen Kohle und Metall). Beschaffung und Produktion werden erschwert durch die Freigabe der Preise für Rohstoffe und Energie. Der Absatz wird behindert durch den Wegfall der staatlichen Abnahmegarantie und durch einen Rückgang der Nachfrage nach Kohle. Durch die Öffnung des russischen Marktes treten außerdem ausländische Anbieter auf, mit denen Malachowka nicht konkurrieren kann. 
In dem Umfang, in dem heute noch produziert und auch abgesetzt werden kann, beeinträchtigt Geldmangel die Produktion. Da auch der Staat als Hauptabnehmer zunehmend in Zahlungsschwierigkeiten gerät, wird die alte Tauschwirtschaft wiederbelebt und teilweise sogar auf die Entlohnung ausgedehnt. Dadurch wird die Auswahl der Zulieferer stark eingeschränkt, so daß das Unternehmen gezwungen ist, auch minderwertige Rohstoffe und Vorprodukte (z. B. von ehemaligen Militärwerken) anzunehmen.

Angesichts dieser Problemlage, die im Sinne unseres Modells die objektive Situation abbildet (vgl. Kästchen 1 in Abb. 2), wurden bisher verschiedene Maßnahmen durchgeführt. Es wird erkannt, daß die Absatzseite heute ein entscheidender Engpaß ist und daß Marketing ein wichtiges neues Instrument darstellt. Auch wurde schon mehrfach versucht, auf andere Produkte (Konsumgüter) umzustellen, was aber (mangels Erfahrung) eher konzeptionslos geschah und zum Teil auch an Imageproblemen (Malachowka versteht sich als traditionsreiches Bergbauunternehmen) scheiterte. Zur Erleichterung der Finanzierung und des Absatzes wurde ein neugegründetes Tochterunternehmen mit der Aufgabe betraut, Absatzmärkte zu erschließen und von den Abnehmern als Bezahlung angebotene Ware weiterzuverkaufen, um so Malachowka Geld zu beschaffen.

Nach Beginn der Perestroika wurden bereits Personalentlassungen vorgenommen (von 1.500 Mitarbeitern auf 900 heute). Ein großes personalpolitisches Problem besteht heute in der hohen Fluktuation der Beschäftigten. Da Malachowka Zahlungsschwierigkeiten hat (zum Zeitpunkt der Befragung waren seit zwei Monaten keine Löhne ausgezahlt worden) und die Verdienstmöglichkeiten im sogenannten "Kommerz" (Handel und private Wachdienste) wesentlich besser sind, ist es sehr schwierig, Leute im Unternehmen zu halten.

\section{Zur Bewertung des Status quo: Vom Regen in die Traufe?}

Um zu erfahren, wie die Befragten (Führungskräfte und Mitarbeiter) ihre persönliche Situation bewerten (vgl. Kästchen 1 in Abb. 2), wurden sie gebeten, aus ihrer Sicht Vor- und Nachteile ihrer heutigen Lebenssituation zu schildern. Da der Transformationsprozeß in mehrfacher Hinsicht einen deutlichen Einschnitt markiert, nahmen wir an, daß die Befragten ihre heutige Situation auch im Vergleich zur Situation vor Beginn der Transformation einschätzen. Deshalb haben wir unmittelbar nach diesem Vergleich gefragt. Nachstehend wird versucht, die Aussagen aus den verschiedenen Interviews in einer Art Bilanz dieses Vergleichs zusammenzufassen (vgl. Abb. 3).

(1) Die heutige Lage ist zum einen dadurch gekennzeichnet, daß viele der früher genossenen Vorteile verlorengegangen sind. Dazu zählt insbesondere das Gefühl der Sicherheit in materieller, sozialer und ideeller Hinsicht. 
Entsprechend wird die Instabiliät und Unsicherheit der heutigen Lage vor allem im Vergleich zu früher als belastend wahrgenommen. Die vom Unternehmen zur Verfügung gestellte soziale Grundversorgung gab die Zuversicht, "daß der nächste Tag relativ ruhig durchläuft"15 oder "daß morgen nichts Schlimmes passiert, daß man nicht gekündigt wird, seine Wohnung behält und die Kinder einen festen Platz behalten" und stärkte das "Vertrauen in die Zukunft"16. Der Leiter eines Versuchzentrums und stellvertretende Generaldirektor illustriert diese Vorteile an einem Beispiel:

"Z. B. früher war alles stabil. Die Leute wußten, was morgen passiert, und was morgen auf sie zukommt, die haben sich keine Sorgen gemacht und haben an ihre soziale Sicherheit geglaubt. Dann erzählt er was konkretes zum Thema: z. $B$. eine neugegründete Familie, jede neugegründete Familie bekam in einem Jahr ein Zimmer und in sechs oder sieben Jahren eine Wohnung. Entsprechend der Anzahl von Familienmitgliedern. Es gab ein Ärztehaus, diverse Erholungsheime... Jetzt folgt wieder die Aufzählung, was Malachowka alles so hatte, und dann wurde die Möglichkeit, diese Erholungsheime zu nutzen, durch den Krieg im Kaukasus beendet."

Heute dagegen werden Instabilität und Zukunftsangst beschrieben. "Die heutige Zeit wird von Unbestimmtheit und Chaos geprägt. Die Leute fragen sich, wohin gehen wir eigentlich?" ${ }^{17}$ Eine Mitarbeiterin aus der Produktion beschreibt die Situation noch etwas krasser: "Sie kann nicht sagen, wo es hingeht. Es herrscht eine Anarchie. Man kann ja nicht begreifen, was heutzutage geschieht."

(2) Gleichzeitig werden aber auch einige Vorteile geschildert, die die heutige Situation im Vergleich zu früher kennzeichnen:

Unter den Pluszeichen der heutigen Situation finden sich vor allem Freiheit und die Möglichkeit, Initiative zu entwickeln. Insbesondere wird als Plus der heutigen Lage die Freiheit im geistigen/ideellen Bereich und in der persönlichen Lebensgestaltung betont. Eine Mitarbeiterin aus der Arbeitsvorbereitung/Konstruktion berichtet:

"Und junge Leute haben auch in der Hinsicht gewonnen, daß sie sich jetzt gönnen können, locker zu leben. Sich locker benehmen. Aus psychologischer Sicht ist es besser, sie verbergen nichts und sie können sich viel erlauben, also bessere Kleider zu tragen. So begreifen sie diese Freiheit: Reisen, gute Kleider, gute Autos und so, genauso wie in der DDR.

Ein Mitarbeiter aus derselben Abteilung "freut sich, daß man sich jetzt amerikanische Filme im Fernsehen anschauen kann, daß man viel lesen kann,

\footnotetext{
${ }^{15}$ Mitarbeiterin der Produktionsabteilung

16 Mitarbeiterin der Abteilung Arbeitsvorbereitung/ Konstruktion

17 Abteilungsleiter der Qualitätsabteilung
} 
daß man sich frei aussprechen kann, daß man jetzt zum Beispiel Sigmund Freud lesen kann und daß man jetzt frei auswählen kann, welche Ideologie man vertritt."

(3) Diese Vorteile werden insbesondere dann als solche erkennbar, wenn man nach den Nachteilen der Situation vor der Perestroika fragt, die heute nicht mehr bestehen. So führte die Überwachung und Bevormundung durch die kommunistische Partei dazu, daß viele nicht "frei sagen konnten, was sie meinten und Angst hatten, verfolgt zu werden"18. Der Abteilungsleiter der Qualitätsabteilung schildert diesen Eindruck in einer Episode:

"Die Leute hatten auch Angst, sich frei zu äußern, dann hat er mir ein Beispiel genannt: Unsere Schauspieler waren mal mit Michael Gorbatschow in den USA gewesen, und eine Schauspielerin wurde gefragt, was ihr so auf Anhieb aufgefallen ist. Und sie meinte, das Beste ist, Kinder haben keine Angst, ihre Gedanken frei zu äußern. Also, keine Freiheit gab `s früher."

Unter diesen Bedingungen dominierte der Eindruck von Bevormundung und Blockierung von Handlungsmöglichkeiten. Der Leiter des Tochterunternehmens geht so weit, zu behaupten, "daß die Russen früher [bis 1984] wie in einem Konzentrationslager gearbeitet haben, also gezwungen waren ohne richtige Bezahlung". Etwas gemäßigter fällt die Äußerung einer Mitarbeiterin aus der Abteilung Arbeitsvorbereitung/Konstruktion aus:

"Ja, wenn man irgendetwas anderes in diesem Unternehmen tun wollte, was anderes, als von oben verordnet wurde, konnte man das nicht machen. Z. B. kann sie sich nicht vorstellen, daß sie plötzlich irgendwelche Verträge mit jemand anderem abgeschlossen hätte, irgendwelche Konsumentenproduktion verabschiedet hätte. Das wäre unmöglich. Also, Wünsche, kreativ zu sein, wurden vorab weggenommen.

Auch vor diesem Hintergrund werden die heutigen Möglichkeiten als positiv eingestuft: "Bestimmend für die neue Mentalität ist Selbstinitiative". ${ }^{19}$ Wie eine Mitarbeiterin aus der Abteilung Konstruktion und Arbeitsvorbereitung schildert, wurden "sowohl die gedankliche Trägheit als auch die unternehmerische Trägheit" überwunden, und heute "ist man kreativ geworden". Der Leiter der Produktionsabteilung sieht diese Initiative als Voraussetzung für Erfolg unter den heutigen Bedingungen: "Die Selbstinitiaitve wurde gefördert, und jeder, der die Marktkonjunktur begriffen hat, kommt durch."

(4) Wie oben angedeutet, sind zwar einige der Nachteile der früheren Lage aufgehoben; es werden jedoch insgesamt heute mehr Nachteile beschrieben,

18 Mitarbeiter der Abteilung Arbeitsvorbereitung/Konstruktion.

19 Abteilungsleiter der Personalabteilung 
weil einerseits viele der früheren Schwierigkeiten erhalten geblieben sind und andererseits mit der Perestroika neue Nachteile hinzugekommen sind:

Einiges was früher als negativ empfunden wurde, wird auch unter den heutigen Bedingungen als unangenehm geschildert. Zur Rolle der "guten Beziehungen" früher äußert ein Mitarbeiter, daß man "entweder Beziehungen haben mußte oder sich begraben lassen konnte" ${ }^{20}$.

Im Vergleich dazu wird die heutige Situation nicht als besser dargestellt. Das Interview mit einem Mitarbeiter aus der Qualitätsabteilung zeigt, daß heute als "gute Beziehungen" bezeichnet wird, was früher "Parteimitgliedschaft" hieß:

(Dolmetscherin): "Ob es früher so war, daß die Karriere nur mit bestimmten politischen Ansichten zu machen war. Ja, das stimmt, nur die Parteimitglieder, also nur die Mitglieder der kommunistischen Partei, waren imstande. Heutzutage zählen keine politischen Ansichten mehr, um Karriere zu machen, sondern gute Beziehungen.

(Interviewerin:) Hat er sich dazu näher geäußert, was das ist? Ist das was ähnliches? (...) Mich interessiert, ob es eine qualitative Veränderung gibt, von Parteimitglied sein bis zu guten Beziehungen heute.

(Dolmetscherin:) Hat sich in Malachowka irgendwas geändert in Sachen Karriere? Gibt es einen qualitativen Unterschied zwischen früher, wo nur ein Parteimitglied eine Karrierechance hatte und heute, wo die Beziehungen zählen? Nein, es gibt überhaupt keinen qualitativen Unterschied. Keine neuen Leute sind hinzugekommen und, er weiß nicht, wie es woanders aussieht, aber in Malachowka ist es so, daß sich nichts geändert hat.

(Interviewerin:) Das heißt, das, was früher die Parteizugehörigkeit war, sind heute die guten Beziehungen. Das ist identisch?

(Dolmetscherin:) Genau, das ist identisch.

Der Leiter des Tochterunternehmens, der früher der Parteisektretär der KPdSU war und an der Parteihochschule studiert hat, berichtet, daß seine heutige Aktivität nur möglich ist auf der Basis früher geknüpfter Beziehungen zu anderen Parteimitgliedern, die heute entscheidende Positionen in der Wirtschaft besetzen:

"Er hat da ganz feste Beziehungen aufgebaut. Und von diesen Beziehungen macht er heute Gebrauch. Weil diese Leute, die früher Parteimitglieder waren und auf einem ziemlich hohem Niveau gestanden haben, wie z. B. ein RegionalParteisekretär oder Bezirks-Parteisekretär, die sind jetzt zu großen Leuten geworden. Die gehören zur Administration."

20 Abteilungsleiter der Produktionsabteilung 
Problematisch ist heute wie früher die Gleichgültigkeit der Leute, wenngleich sich die Ursachen der Gleichgültigkeit geändert haben:

"Früher herrschte sie deswegen, weil man wußte, daß man nicht mehr Geld dazu kriegt, heute deswegen, weil man kein Geld dazu kriegt, weil man überhaupt nicht weiß, was zu tun ist, weil diese Ziellosigkeit im Lande herrscht." 21

Zugenommen hat im Vergleich zu früher das Mißtrauen gegenüber Autoritäten. Stellvertretend für viele ähnliche Äußerungen stehen die Ansichten eines Mitarbeiters aus der Arbeitsvorbereitung/Konstruktion über Politiker:

(Dolmetscherin:) "Also, ich habe ihn gefragt, was wäre, wenn er ein Politiker wäre. Er meinte, Politiker sind alle faule Äpfel. Er will auf keinen Fall ein Politiker werden, er will damit gar nichts zu tun haben, die sind alle schmutzig, die sind Räuber, und dann hat er angefangen, die zu beschimpfen. Dann hat er sehr viele Beispiele erwähnt, die allerdings aus Zeitungen und aus den Fernsehsendungen kommen, was die so alles geklaut haben.

(Interviewerin:) Die Politiker?

(Dolmetscherin:) Die Politiker. Auch die Politiker, die jetzt von der Politikbühne weggegangen sind, welche Häuser und wo sie sich auch, sie sich diese Häuser aufgebaut haben, daß die meisten auch nach Amerika und in andere Länder gegangen sind.

(Interviewerin:) Ausgewandert?

(Dolmetscherin:) Ja, ausgewandert. Die haben russische Staatsangehörigkeit erhalten, aber leben woanders. Die haben ihr Geld, die haben alles, was die wollten, und Leute haben sie grausam betrogen. Und deswegen kann er keinem sein Vertrauen schenken und will auch keine Leute betrügen, weil jeder, der an die Spitze rankommt, dann nur irgendwie das Geld in seine Tasche reintut und kann auch nichts ändern, also er sieht überhaupt keinen Ausgang und so.

(Interviewerin:) War das früher anders? Hat er das gesagt? Also sind die heute besonders korrupt?

(Dolmetscherin:) Er meinte, daß, vielleicht, war früher auch da oben irgendwas los, er weiß es nicht. So, bloß, bloß er konnte auch leben, er konnte sich wenigstens, er konnte wenigstens seine Mindestbedürfnisse befriedigen, und jetzt kann er das nicht.

(Interviewerin:) Also glaubt er, daß die Politiker heute schlimmer sind als früher?

21 Mitarbeiter der Qualitätsabteilung 
(Dolmetscherin:) Ja, auf jeden Fall. Das sind keine Politiker, das sind nur Räuber."

(5) Durch den Transformationsprozeß zusätzlich entstandene Schwierigkeiten sind insbesondere materielle Not, wachsende Kriminalität und zunehmende soziale Unterschiede. Der heute gravierendste und neue Nachteil, der in allen Interviews zum Ausdruck kam, besteht in der unzureichenden materiellen Versorgung. Die Abteilungsleiterin aus der Materialbeschaffung erläutert:

"Ihre Geldsituation war besser und stabiler, mit 130 Rubel im Monat hat sie in ihrem Kühlschrank mehr Lebensmittel gehabt als jetzt mit einer Million."

$\mathrm{Zu}$ der beschriebenen Geldnot kommt eine wachsende Kriminalität: "Die Kriminalität hat rapide zugenommen, sie hat höllische Angst um ihre Kinder."22

Durch die undurchschaubare Lage sind auch heute die Möglichkeiten, Geld zu verdienen, vielseitiger und unüberschaubarer geworden. Von den Befragten wird vor allem beklagt, daß heute die Unterschiede zwischen Armen und Reichen größer sind als vorher. Eine Mitarbeiterin aus der Qualitätsabteilung beschreibt diesen Eindruck folgendermaßen:

"Dann hat sie eine Unterteilung getroffen, und zwar früher konnte man unterscheiden: Es gab Leute, die gut gelebt haben, das war eventuell die Parteispitze, die hatten Geld, Wohlstand. Es gab sehr viele Leute, das war die Mehrheit, die mittelmäßig gelebt haben, und es gab Alkoholiker, die gar nichts gehabt haben oder faule Leute, die nichts getan haben und deswegen auch nichts verdient haben. Heutzutage kann man Leute auf zweierlei Lager verteilen: Die Masse, die gar nichts hat und die Kriminellen und Kommerzleute, bei denen Betrug zu einem inneren Wert wird."

Stellt man abschließend zusammenfassend die Frage, wie sich die heutige Situation im Vergleich zu dem Stadium vor Beginn der Transformation darstellt, so ergibt sich eine Bilanz, in der vor allem Verluste (von Vorteilen der früheren Situation) und neu entstandene Schwierigkeiten überwiegen.

Der heutige Zustand zwischen der geschlossenen Gesellschaft vor Beginn der Transformation und der offenen Gesellschaft wird demnach in mehrfacher Hinsicht als problematisch erlebt. Einerseits werden viele Vorteile vermißt, die charakteristisch sind für geschlossene Gesellschaften (Gebert/ Boerner 1995); andererseits stellen sich typische Vorteile des offenen Musters (z. B. Freiheit) aus der Nähe betrachtet auch als belastend heraus (z. B. Orientierungslosigkeit). Hinzu kommt, daß einige der erhofften Vorteile des offenen Musters noch nicht erreicht wurden und daher zu Enttäuschungen führen: "Eine große Hoffnung war die Aussicht, daß ihr Wohlstand verbessert wird. Und dann wurde sie aller-

22 Abteilungsleiterin der Materialbeschaffung 
dings etwas enttäuscht, weil sie auf keinen Fall mehr Geld bekommt." ${ }^{23}$ Aus diesem Grund können selbst die bereits erreichten Verbesserungen wie z. B. die bessere Versorgung mit Konsumgütern nicht vollständig genutzt werden.

Abbildung 3: Kennzeichen der heutigen Situation "Dazwischen" in bezug auf ihre Vor- und Nachteile im Vergleich zu früher.

Merkmale der heutigen Situation

(1) verlorene Vorteile

soziale Sicherheiten (Wohnungen,

Gesundheitsversorgung,

Kindertagesstätten, Frei-

zeiteinrichtungen etc.)

materielle Sicherheiten

Stabilität, Orientierung und Vertrauen

\section{(3) verlorene Nachteile}

Freiheitsbeschneidungen, Überwachung und Bevormundung, Angst vor Verfolgung und staatlicher Willkür

\section{(2) neue Vorteile}

Freiheit in der Lebensgestaltung und in geistiger/ ideeller Hinsicht, Zugang zu ausländischen Konsumgütern, Initiative

\section{(4) gebliebene Nachteile}

Abhängigkeit von Beziehungen

Gleichgültigkeit, verstärktes Mißtrauen

\section{(5) neue Nachteile}

Kriminalität, Korruption,

Räubermentalität

Orientierungslosigkeit

materielle Not, Arbeitslosigkeit

zunehmende soziale Unterschiede

"Tja, vielleicht der einzige Pluspunkt ist, daß es sehr viele Konsumgüter gibt. Obwohl, jede Medaille hat zwei Seiten: Die meisten Konsumgüter, die heutzutage verkauft werden, besonders die ausländische Produktion, sind schlecht. Sie sind von vorneherein verdorben, und sie bemüht sich, inländische Produktion zu kaufen. Aber das fällt nicht so leicht. Unsere Wirtschaft wird

${ }^{23}$ Mitarbeiterin der Produktionsabteilung 
nicht gefördert, man spricht davon, daß die Inflation zurückgegangen ist, aber das stimmt nicht, wenn man die Situation objektiv betrachtet. Der Wohlstand der Menschen ist zurückgegangen, und deswegen können auf die Preise nicht mehr Prozent aufgeschlagen werden als es früher war. Sie hat im Laufe dieses Transformationsprozesses nichts besseres erfahren." 24

Schließlich führen die mit der Umgestaltung verbundenen Friktionen dazu, daß der Zustand des Dazwischen nicht lediglich als ein Tausch von spezifischen Vor- und Nachteilen erlebt wird. Diese Reibungsverluste sind insbesondere auf zwei Eigentümlichkeiten des russischen Transformationsprozesseß zurückzuführen.

(1) Der Ausstieg aus der geschlossenen Gesellschaft wurde (anders als in der ehemaligen DDR) mit den Mitteln und von den Akteuren der geschlossenen Gesellschaft vollzogen und gewissermaßen als Reform von oben (Dittrich 1992; Staniszkis 1991) verordnet. Eine Mitarbeiterin der Organisationsabteilung schildert ihre Erinnnerung in einem Bild:

"Als das Ganze angefangen hat, war die Mentalität der Leute nicht reif für diese Umgestaltung. Leute wurden wie blinde Kätzchen oder Hündchen ins Wasser geworfen. Betriebe wurden zugemacht, und darauf war auch keiner vorbereitet. Die Leute wußten nicht, was sie jetzt anfangen sollen."

(2) Die Ziele des gesellschaftlichen Wandels sind unklar (Stojanov 1992). Während ein großer Teil der Bevölkerung als Zielvorstellung eine Variante des Modells der offenen Gesellschaft anstrebt ("Vielleicht nähern wir uns langsam der zivilisierten Welt" ${ }^{\prime 25}$ ), ist unklar, ob die politischen Akteure ebenfalls dieses Ziel anstreben. Die Befragten vermuten vielmehr, daß die heute herrschende "alte Nomenklatura" weniger an Demokratie und Marktwirtschaft als an einer Vermehrung ihres persönlichen Wohlstandes interessiert ist. Diese Haltung zeigt sich z. B. in dem Interview mit einer Mitarbeiterin der Organisationsabteilung:

(Dolmetscherin:)"Es muß eine neue Mannschaft kommen, eine neue Präsidentenmannschaft. Es reicht nicht, einen Präsidenten zu ersetzen, es bringt auch nichts. Aber eine neue Mannschaft kann also Leute mitreißen, daß einer dem anderen erzählt, so machen wir es, und wir kriegen dann Unterstützung und so. Daß alle es zusammen machen. Ja, und so lange, wie da diese alte Mannschaft sitzt, an der Spitze, ist nichts zu unternehmen. Zum Beispiel, vor kurzem wurde erklärt und verkündet, daß jetzt das Geld für den TschetschenienAufbau aufgebracht werden muß. Wie denn, da herrscht immer noch der Krieg, und alles, was reininvestiert wird, wird sofort auch zerstört. Man wollte nur auf

\footnotetext{
24 Mitarbeiterin der Organisationsabteilung

25 Abteilungsleiter der Qualitätsabteilung
} 
die Art und Weise an das Geld rankommen, daß das Geld in die Tasche, in jemandes Tasche letzten Endes landet. Was jetzt passiert, ist ein Prozeß der Zerstörung, und es wird nichts aufgebaut, so generell gesehen. Als Beispiel hat sie Erholungsheime, das Ärztehaus von Malachowka aufgezählt, Kindergärten, die jetzt alle abgeschafft wurden oder jemandem anderen übergeben. Und ein Aufbauprozeß, das muß ein top-down-Prozeß sein, also die Initiative muß von oben kommen, und dann muß die neue Mannschaft landesweit auftreten...

(Interviewerin:) Das gilt nicht nur für Malachowka, sondern für ganz Rußland?

(Dolmetscherin:) Ich habe sie nochmal daran erinnnert, daß sie meinte, Malachowka ist Rußland im Kleinformat, ja, und dann fragte ich sie, was kann denn die Unternehmensleitung unternehmen, damit es besser wird? Sie meinte: gar nichts, weil es eben ein top-down-Prozeß ist. Und solange an der Spitze, an der Regierungsspitze, irgendjemand bleibt, der die ganze Sache lähmt, ist unten nichts zu unternehmen. Es muß an jeder Stufe alles neu gestaltet werden und alles umstrukturiert werden. Von der Spitze bis zur Stadtverwaltug, bis zur Regionalverwaltung usw. und so fort. Und dann, momentan hat die Unternehmensleitung keine Möglichkeit, etwas zu ändern oder etwas besseres zu machen. So, Fisch verdirbt auch vom Kopf.

(Interviewerin:) Wie, nochmal. Ach so, der Fisch verfault vom Kopf her.

(Dolmetscherin:) Genau."

Die persönliche Bilanz dieses Vergleiches früher-heute wird also in der Regel negativ ausfallen. Angesichts der heutigen Schwierigkeiten verwundert es nicht, daß heute vor allem die Vorteile der alten Ordnung vermißt werden. Allerdings konnten wir feststellen, daß die Befragten sich dennoch kein Zurück zu dem Zustand vor der Perestroika wünschen, sondern auf dem begonnenen Weg weitergehen möchten. Eine Mitarbeiterin aus der Abteilung Arbeitsvorbereitung "meint, daß auch die Präsidentenwahl es ganz gut gezeigt, ganz kraß das gezeigt hat, daß keiner ein Come-back für zwei Rubel zwanzig Wurst möchte. Also, Wurst hat früher zwei Rubel zwanzig Kopeken gekostet, so, und Leute wollen das Come-back nicht mehr erleben." Verständlich wird diese Tatsache, wenn man die Reaktionsweisen der Befragten auf die heutige Situation näher bleuchtet.

\section{Handlungsoptionen im Status quo: Handlungsverzicht oder Initiative?}

Vor dem Hintergrund der berichteten Interviewausschnitte verdichten sich die Hinweise darauf, daß die heutige Lage mehrheitlich als eine Bedrohung im Sinne von Lazarus wahrgenommen wird (vgl. Kästchen 2 in Abb. 2). Nicht nur werden die existentiellen Bedrohungen materieller und ideeller Art (Orientierungslosigkeit, Verlust von Geborgenheit und Zuversicht) genauer 
benannt; auch werden zusätzlich enttäuschte Hoffnungen im Hinblick auf den Transformationsprozeß artikuliert. Speziell für das untersuchte Unternehmen Malachowka läßt sich die aktuelle Lage objektiv wie oben beschrieben konkretisieren: Es fehlt an Geld, an Aufträgen, an Absatzmöglichkeiten, an Rohstoffen und Material, an Energie und an qualifizierten Mitarbeitern.

Nachstehend wird beschrieben, wie sich Menschen in Rußland angesichts dieser Bedrohung verhalten, welche Handlungsmuster sie also zur Bewältigung dieser Situation präferieren. Zusätzlich sollen anhand der Befragung die Handlungspräferenzen erklärt werden. Dabei wurde uns nicht nur das Handeln der Befragten selbst geschildert, sondern häufig auch Verhaltensweisen anderer Personen. Die von den Befragten berichteten Strategien zur Bedrohungsbewältigung lassen sich in das dargestellte Modell einordnen, das sich an das Streßbewältigungsmodell von Lazarus anlehnt.

\subsection{Handlungsverzicht als erlerntes Verhaltensmuster}

Als das heute vorherrschende Muster wurde uns eine passive Haltung der Mitarbeiter berichtet (vgl. Kästchen A in Abb. 2). Dieser Handlungsverzicht ist zunächst dadurch zu erklären, daß die Beteiligten weder zu früheren Zeiten noch unter den heutigen Bedingungen den Eindruck haben, über ausreichend Situationskontrolle zu verfügen. War vor Beginn des Transformationsprozesses systembedingt wenig Spielraum für eigene Entscheidungen und Handlungen vorhanden, was sich z. B. auf der betrieblichen Ebene dadurch zeigte, daß Eigeninitiative teilweise sogar bestraft wurde (Puffer 1994), scheinen unter den oben skizzierten heutigen Rahmenbedingungen (volkswirtschaftliche Restriktionen, Restriktionen des Unternehmens, autoritäre Führung) eigene Initiativen auch nur eingeschränkt möglich. Bestand bei allen Befragten im wesentlichen Einigkeit über unzureichende Situationskontrolle, so fanden wir doch zwei entgegengesetzte Muster, diese Tatsache mental zu verarbeiten. Die Gemeinsamkeit beider Muster liegt auf der Handlungsebene in einem Handlungsverzicht, während die Erklärung dieser Verhaltensweise durch psychologische Verarbeitungsprozesse unterschiedlich ausfällt. Da uns für den weiteren Verlauf des Transformationsprozesses die Ursachen des Handlungsverzichts bedeutsam erscheinen, seien beide Erklärungsmuster nachstehend erläutert.

(1) Von einzelnen Befragten wurde uns eine Reaktionsweise geschildert, die sich als Resignation bezeichnen läßt. Diese Resignation kommt dadurch zustande, daß die Befragten angesichts der bedrohlichen Situation (1. Bewertung) und der Tatsache, selbst keine Situationskontrolle zu besitzen (2. Bewertung), um diese Situation zu bewältigen, in ihrem Bewußtsein die Differenz zwischen dem erwünschten Soll-Zustand und dem täglich erlebten IstZustand dadurch reduzieren, daß sie den Sollwert senken und ergänzend den IstWert durch Beschönigung anheben. Diese Befragten sind davon überzeugt, daß 
sich ohnehin nichts an der bedrohlichen Lage ändern wird und finden sich subjektiv mit dieser Tatsache ab, indem sie vor allem ihr Anspruchsniveau senken. Durch diese Form der Bewältigung der bedrohlichen Lage, die Lazarus als intrapsychische Anpassung bezeichnet, wird nicht nur die Differenz zwischen Soll- und Ist-Wert eingeschmolzen, sondern in der Folge auch die eigene Handlungsbereitschaft reduziert, da aus subjektiver Sicht kein Handlungsbedarf mehr besteht.

Ein Beispiel für den so zustandegekommenen Handlungsverzicht beschreibt eine Mitarbeiterin der Qualitätsabteilung. Sie "meinte, es gibt mehr Resignatoren, weil die Leute, naja, eigentlich Angst haben, na ja, nicht eigentlich Angst haben, weil sie nicht glauben, daß sich irgendwas ändert. Alleine ist es nicht zu schaffen." Wie uns berichtet wurde, ist diese resignative Form der Passivität allerdings keineswegs vorherrschend, sondern nur für eine kleinere Gruppe von Mitarbeitern kennzeichnend, vor allem für unflexible Mitarbeiter, also ältere, schlecht ausgebildete und Frauen.

(2) Von der Mehrheit der Befragten wird der Verzicht auf Aktivität auf andere Weise erklärt als durch intrapsychische Anpassung im Sinne von Lazarus. War das Muster "Resignation" dadurch erklärbar, daß jede Hoffnung auf eine Verbesserung der Lage aufgegeben wurde, ist die häufigere Art der Handlungsblockierung im Gegenteil durch eine Hoffnung gekennzeichnet, die zum Teil sogar utopisch übersteigert erscheint. In der Sprache von Lazarus (1991) bleibt bei dieser Variante der Sollwert im Bewußtsein der betreffenden Person erhalten. Damit bleibt auch die Soll-Ist-Differenz im Bewußtsein der Akteure erhalten, mithin also ein potentieller Leidensanlaß, und man kann insofern fragen, warum nicht auch in dieser Stichprobe der Weg der intrapsychischen Anpassung beschritten wird, den Lazarus für diese Konstellation erwartet: die subjektive Einschmelzung der Soll- Ist-Differenz.

Stattdessen sehen wir ein Aushalten dieser Differenz, was sich daran zeigt, daß die Akteure abwarten. Zusätzlich ist dieses Abwarten insofern bemerkenswert, als die Befragten gleichzeitig erhebliche Hoffnungspotentiale äußerten. Ein solcher Handlungsverzicht trotz Hoffnung wird als ein erlerntes Verhaltensmuster speziell der russischen Befragten verständlich. So haben die Befragten anscheinend durch ihre Erfahrungen aus dem Zeitraum vor der Transformation bereits gelernt, generell mit einer Abweichung zwischen Sollund Ist-Wert zu leben, wobei sie auch durch die ständige Betonung des anvisierten Soll-Zustandes (sozialistisch-kommunistisches Gesellschafts-Ideal) durch systemimmanente Propaganda kaum Gelegenheit hatten, diese Differenz zu verdrängen. Hinzu kommt, daß auch die Erfahrung von wenig Situationskontrolle eine historische ist, die sich offensichtlich auf die Erwartungsstruktur der Menschen ausgewirkt hat: "Das russische Volk hat immer einen Zaren gebraucht, um gut zu arbeiten", und daher ist es dem 
"russischen Volk so immanent, immer eine feste Vorgabe zu bekommen". ${ }^{26}$ Beide Aspekte werden in dem folgenden Interviewausschnitt deutlich:

" Das russische Volk ist auch sehr geduldig und wartet immer darauf, daß die Regierung irgendwann mal weiser wird. In anderen Ländern wird das nicht geduldet. (...) Es ist ein Teil der russischen Seele, zu glauben, daß es besser wird, aber wir tun es nicht, es tut die Regierung, die irgendwann mal weiser wird, und die uns dann beschert mit den schönen Sachen des Lebens. Und sie meinte, daß sich keiner alleine durchsetzen kann. Deswegen, wenn einer eine schöne Idee hat, aber keine Unterstützung, dann setzt er sich lieber zur Ruhe, weil es ansonsten zu keinem Ergebnis führt. Und in dieser Hinsicht ist es heute noch schlimmer geworden als früher. ${ }^{27}$

Da Soll-Ist-Wert-Abweichungen historische Realität waren, hat "das russische Volk" gelernt, mit einer Utopie zu leben, deren Einlösung jedoch aufgrund ebenfalls historischer Erfahrungen von wenig Situationskontrolle von "höheren Mächten" (dem Zaren, der Regierung, den Vorgesetzten etc.) erwartet wird (Stojanov 1992; Staniszkis 1991; Puffer 1994), so daß eine Soll-IstAbweichung weder unmittelbar zum Handeln noch zur intrapsychischen Anpassung führt. Es liegt hier die Frage nahe, ob dieses Muster nicht eine generelle Strategie geschlossener Gesellschaften darstellen könnte, die Leidensfähigkeit der Menschen zu stabilisieren.

Zur Illustration dieser Hoffnung sei abschließend erwähnt, daß die gegenwärtigen Probleme in der Wahrnehmung vieler Befragten lediglich die einer Übergangsphase sind. Die Zuversicht, daß Rußland seinen eigenen Weg souverän und erfolgreich gehen wird, stammt teilweise aus dem nationalen Selbstbewußtsein und dem Glauben an die Fähigkeiten des russischen Volkes (Geduld, Kraft und Leidensfähigkeit) und wird teilweise aus dem Vergleich mit der Geschichte westlicher Staaten abgeleitet (USA und Deutschland), die ebenfalls schwierige Zeiten erlebt haben. Angesichts der russischen Realität stellt sich jedoch die Frage, inwiefern diese Variante der Erzeugung von Hoffnung in den Bereich der Utopie einzuordnen ist, die subjektiv das Aushalten der gegenwärtigen Schwierigkeiten ermöglicht.

In dieser Hoffnung liegt vermutlich auch der Grund dafür, daß die Befragten sich trotz der überwiegend als nachteilig bzw. bedrohlich erlebten Situation kein Zurück in die Zeit vor der Perestroika wünschen. Dieses Zurück, das immerhin eine Art von (wenn auch rückwärtsgewandter) Initiative erfordern würde, wird von allen Beteiligten abgelehnt.

\footnotetext{
26 Mitarbeiter der Qualitätsabteilung

27 Mitarbeiterin Qualitätsabteilung
} 


\section{2. "Angriff" in Form konstruktiver Initiative}

Dieses Verhaltensmuster fanden wir vergleichsweise selten (vgl. Kästchen B in Abb. 2). Allem Anschein nach können sich nur diejenigen positive Initiative leisten, deren finanzielles Auskommen anderweitig (durch Familie, Ehepartner) gesichert ist. Damit ist allerdings die individuelle Bedrohung bereits reduziert. Dieser Fall trifft z. B. auf den Leiter der Produktionsabteilung zu:

(Dolmetscherin:) "Da meinte er, er tut das heutzutage, das ist seine, das ist sein Lieblingsjob, und nur deswegen arbeitet er hier. Von seinem Geld, das er hier kriegt, kann er nicht so gut leben.

(Interviewerin:) Womit ist er denn zufrieden?

(Dolmetscherin:) Ehm, damit, was er hier tut, also Leute leiten, Produktion führen und so. Das macht ihm Spaß.

(Interviewerin:) Das Ideelle ist mehr wert...

(Dolmetscherin:) Routine, eben diese Routine, die macht ihm Spaß. Das ist sein Lieblings-Job. (...) Ja, also seine Frau hat genügend Geld, und deswegen kann er, und deswegen kann er sich gönnen, die Arbeit zu machen, die ihm Spaß macht, eh und Geldeinbußen mit einkalkulieren. "

Was in Malachowka bisher an positiver Initiative geleistet wurde und wird, ging zumeist von der Unternehmensleitung oder dem mittleren Management aus, wie es z. B. der Leiter der Produktionsabteilung beschreibt:

(Dolmetscherin:)" Die Meister praktisch, die Abteilungsleiter sind positive Initiatoren. Dann meinte er, diese Abteilungsleiter haben dann diese Funktion, Werkstattleiter oder Meister oder bzw. auch Arbeiter in bestimmte Entscheidungssituationen reinzuzwingen.

(Interviewerin:) Also, aus der Resignation 'ne positive Initiative zu machen?

(Dolmetscherin:) Ja, genau."

Die Initiative bestand z. B. darin, versuchsweise auf Konsumgüter umzustellen, ein Qualitätsmanagement einzuführen oder Mitarbeiter umzuschulen. Wie die Befragten berichteten, erwiesen sich diese Versuche jedoch überwiegend als erfolglos.

\section{3. "Flucht" in zwielichtige Initiative}

Diese Möglichkeit läßt sich verständlicherweise schwer direkt abfragen, wurde uns aber unter anderem durch indirekte Hinweise auf das Verhalten anderer als eine im Augenblick häufig anzutreffende und aussichtsreiche Strategie dargestellt. Tradition hat auch diese Strategie insofern, als Schattenwirtschaft auch unter den Bedingungen des alten Systems nicht unbekannt war (Götz 1991). "In der Regel war das, was effizient war, illegal" (Hentze/ Lindert 1992: 247). Nahezu die einzige Möglichkeit, Geld zu verdienen, besteht auch heute 
darin Handel zu treiben, wobei sich dieser Handel ganz offensichtlich am Rande der Legalität bewegt.

Eine Form dieser Art von Aktivität führt zu dem oben beschriebenen Phänomen der Fluktuation und wäre damit im Sinne von Lazarus als "Flucht" aus dem Unternehmen zu bezeichnen (vgl. Kästchen C in Abb. 2). Diese Art von Initiative ergreifen vor allem jüngere, gut ausgebildete und flexible Mitarbeiter. Dies trifft das Unternehmen Malachowka umso härter, als eine derartige Mobilität der Mitarbeiter neu ist und Führungskräfte daher nicht darauf vorbereitet sind.

Die früheren Bindungsmechanismen, die Fluktuation weitgehend unterbunden haben, stehen heute nicht mehr oder nur noch sehr eingeschränkt zur Verfügung. Unter sowjetischen Bedingungen besaß das Unternehmen das Monopol auf fast alle Sozialleistungen (Wohnungen, Kindergartenplätze, Ferienplätze, Freizeiteinrichtungen, Krankenversorgung), so daß sich eine gewissermaßen natürliche Abhängigkeit der Belegschaft ergab. Außerdem bestand eine allgemein anerkannte Norm, nach der ein Unternehmenswechsel prinzipiell als Verrat und Treuebruch gewertet wurde. Heute empfinden nur noch Ältere eine starke Bindung an das Unternehmen, wie z. B. ein stellvertretender Direktor (Ressort Wirtschaft):

Dann, eh, hat er allerdings auch erwähnt, daß die meisten hier fünfzehn bis zwanzig Jahre gearbeitet haben, und allerdings, die fühlen sich erst aus dieser Hinsicht verantwortlich. Also, die empfinden das ganze als ihr Zuhause. Die Belegschaft ist ihre Familie, und naja, dann fühlt man sich halt verantwortlich, obwohl man es gar nicht ist. Man möchte auch besser sein, es gehört auch zum Image.

Eine interessante Variante, die auch darauf hindeutet, daß die Grenzen zwischen "positiver" und "negativer" Initiative fließend sind, ist eine Art "Flucht auf Zeit", die uns als fluktuationshemmende Maßnahme geschildert wurde. Mitarbeiter, die die Absicht äußern, das Unternehmen zu verlassen, erhalten die Möglichkeit, unentgeltlichen Urlaub zu nehmen und in dieser Zeit (entgeltlich!) in einer sogenannten "Kommerzeinrichtung" zu arbeiten, und werden danach wieder zu den alten Konditionen im Betrieb eingestellt. Der Leiter der Produktionsabteilung berichtet:

(Dolmetscherin:) "Und die versuchen, junge Mitarbeiter mit aller Kraft zu überreden, und es werden auch diverse Tricks eingesetzt, z. B. wenn irgendjemand aus dem Grunde kündigt, daß er zu wenig Geld hat, dann schlägt er vor, er nimmt zwei, drei Monate Urlaub, ohne finanzielle Unterstützung und geht irgendwo hin.(...) Er läßt entweder schuften, büffeln oder irgendwelche Geschäfte tätigen, und dann kommt der Mensch zurück, nachdem er etwas Geld eingesammelt hat.

(Interviewerin:) Also Incentives? 
(Dolmetscherin:) Ja, das ist eben ein Kompromiß. Aber es ist unentbehrlich, diese Kompromisse einzugehen, weil ansonsten diese Mitarbeiterfluktuation noch größer wäre.

(Interviewerin:) Gibt es denn woanders so viele bessere Möglichkeiten, also gibt der Arbeitsmarkt das her, daß man woanders viel leichter einen Job findet?

(Dolmetscherin:) In diesen Kommerzeinrichtungen gibt es manche Möglichkeiten, wie man sich als Wachdienst bewirbt ...

(Interviewerin:) Ach ja, stimmt, genau, das hatten wir.

(Dolmetscherin:) ... oder, genau, wie man eben diese Schiebereigeschäfte tätigt. Im Ausland einkaufen, hier verkaufen."

Allerdings bewirkt diese Maßnahme zuweilen auch das Gegenteil, nämlich dann, wenn die Konditionen im Unternehmen Malachowka so schlecht sind, daß die Mitarbeiter endgültig den "Kommerz" vorziehen.

Der Leiter der Produktionsabteilung nennt auch die Möglichkeit, die halblegale Energie der Mitarbeiter unmittelbar für das Unternehmen nutzbar zu machen:

(Dolmetscherin:) "Er kann auch diese Resignatoren und diese negativen Initiatoren umwandeln, indem er bestimmte Vorschläge macht bzw. Druck ausübt. Aber diejenigen, die, naja, die negativen Initiatoren, die unverbesserlich sind, denen muß man schon etwas Freiheit gönnen, indem sie ihre positive Funktion auch ausüben, vielleicht können sie auf diesem krummen Wege auch Geld für das Unternehmen beschaffen, indem sie ihre krummen Geschäfte betreiben.

(Interviewerin:) Ach so, also, den Leuten....

(Dolmetscherin:) Den Leuten...

(Interviewerin:) Es gibt manche Leute, denen muß man mit Druck begegnen, um sie dann zu negativen ...

(Dolmetscherin:) Genau, meistens Resignatoren. Es gibt negative Initiatoren, die bloß etwas Druck brauchen...

(Interviewerin:) Und werden dann positiv?

(Dolmetscherin:) Und werden dann positiv, genau. Und diejenigen, die sich nicht umstylen lassen, die können auch weggehen, und vielleicht sie werden dann ihre eigene Produktion gründen."

Auch innerhalb der Unternehmung sind die Grenzen zwischen positiver und negativer Initiative nicht eindeutig zu bestimmen. Nach Einschätzung der Befragten sind sowohl Politiker als auch die Unternehmensleitung an verschiedenen Formen zwielichtiger Initiative beteiligt, und dies wird in der gegenwärtigen Situation als einzige Möglichkeit wahrgenommen, das 
Unternehmen über Wasser zu halten. Eine Mitarbeiterin aus der Abteilung Arbeitsvorbereitung/Konstruktion berichtet:

(Dolmetscherin:) "So, sie hat selber angefangen mit Flexibilität. Ach ja, Zwischentöne, ich hab versucht, herauszufinden, erstmal, welche Leute sie als Zwischentöne bezeichnet.

(Interviewerin:) Welche Leute?

(Dolmetscherin:) Ja, genau, und sie hat mit einer Situation und dem Unternehmen insgesamt angefangen, daß sie das Verhaltensmuster des Unternehmens, sprich des Vorgesetzten, ähm, beziehungsweise der Leiter, als zwischentönig betrachtet.

(Interviewerin:) Zwischen Initiative und Resignation? Oder zwischen positiver und negativer Initiative?

(Dolmetscherin:) Nein. Äh, zwischen positiv und negativ, darauf wollte ich nochmal hinweisen. Und ihretwegen ist es ein Beweis der Flexibilität. Diese Flexibilität ist dazu notwendig, um sich über Wasser zu halten. Und deswegen läßt es sich nicht eindeutig beurteilen, ob es gut oder schlecht ist, was, äh, die Unternehmensleitung macht.

(Interviewerin:) Also man braucht ein paar schräge Geschäfte, um zu überleben?

(Dolmetscherin:) Genau! Paß auf, und jetzt kommen wir auf die krummen Geschäfte der Unternehmensleitung. So! Es wurden manche Konsumgeschäfte eröffnet, die stillschweigend dem Unternehmen angehören. Und letztens haben sich Leute gewundert, warum das Geld nicht von der Bank kommt, sondern irgendwie schon in Gebrauch war. So, und zwar, dieses Geld ist durch Geschäfte entstanden, äh, vielleicht durch Produktion, irgendwelche linke Produktion, die in diesen Geschäften verkauft wurde. Ähm, naja, aber was können sie sonst tun? Die müssen doch Leute irgendwie über Wasser halten, und der Staat weigert sich, das Geld rechtzeitig zu bezahlen.

(Interviewerin:) Also die machen krumme Geschäfte und bezahlen davon ihre Mitarbeiter?

(Dolmetscherin:) Ja, aber nicht immer. Meistens geht dieses Geld, um irgendwelche Transporte zu tätigen, und um die Produktion am Leben zu erhalten.

Ein konkretes Beispiel für den fließenden Übergang zwischen positiver und negativer Initiative ist das neugegründete Tochterunternehmen. Während der Leiter des Tochterunternehmens sein Verhalten als Ausdruck einer besonderen Flexibilität bezeichnet, bewertet ein Mitarbeiter aus der Abteilung Konstruktion/Arbeitsvorbereitung diese Initiative anders: 
"Zum Beispiel, der Generaldirektor oder irgendwelche Führungskräfte, die haben ja auch Zusatzeinkünfte von ihren Tochterunternehmen. Was heißt das? Das heißt, die wickeln irgendwelche krummen Geschäfte ab durch die Tochterunternehmen, und deswegen bekommen sie auch ihr Geld."

\subsection{Zusammenfassung}

Faßt man die Handlungsoptionen bzw. die Reaktionen auf die heutige Umbruchsituation zusammen, so kann man feststellen, daß die Situation mehrheitlich als bedrohlich empfunden wird. Da die Erfahrung von wenig Situationskontrolle nicht neu ist und Abwarten ein tradiertes russisches Reaktionsmuster darstellt, ist heute Handlungsverzicht die am meisten verbreitete Reaktion auf die aktuellen Bedingungen. Dieser Handlungsverzicht ist nur zum Teil durch resignative intrapsychische Anpassungen zu erklären; dominierend ist vielmehr ein Abwarteverhalten, das sich mit einer diffusen Hoffnung auf eine Verbesserung der Situation verbindet. Nur bei wahrgenommener Situationskontrolle (meist auf den höheren Hierarchiestufen oder unter verminderter Bedrohung aufgrund materieller Absicherung) ist positive Initiative ("Angriff") anzutreffen, die im Bewußtsein der Akteure zudem nicht oder kaum durch Erfolg verstärkt wird, da sie bisher meistens erfolglos blieb. Vor diesem Hintergrund wird die Initiative in zwielichtige Bahnen gelenkt und insofern durch Erfolg verstärkt, als sie teilweise als die einzige Überlebensmöglichkeit gilt. Dabei sind sowohl de facto als auch in der Bewertung der Befragten die Grenzen zwischen positiver und negativer Initiative fließend. Während die Unternehmensleitung selbst zwischen positiver und negativer Initiative laviert, führt bei vielen flexibleren Mitarbeitern (insbesondere jüngeren, gut ausgebildeten) negative Initiative zu einer Flucht aus dem Unternehmen. Die Folge für Malachowka ist eine Selbstselektion der Beschäftigten, die für das Unternehmen äußerst ungünstig ist: Es gehen vor allem die jüngeren und gut ausgebildeten Mitarbeiter, während sich die älteren und schlechter ausgebildeten an ihren Arbeitsplatz klammern.

\section{Führung als Drahtseilakt: Randbedingungen und Handlungsoptionen}

\subsection{Ungünstiges Betriebsklima: Konflikte und Mißtrauen}

Bevor überlegt wird, was Führungskräfte angesichts der skizzierten Lage tun können, soll hier zur Vervollständigung der Status quo-Beschreibung ein Aspekt vorangestellt werden, der im Laufe der Interviews auffiel. Sowohl im Umgang der Mitarbeiter untereinander als auch im Verhältnis zu Führungskräften ist im untersuchten Betrieb ein auffälliges Mißtrauen vorherrschend. 
Mißtrauen unter den Kollegen entsteht zum einen aufgrund der Verschärfung persönlicher Probleme im Alltag, die zu Spannungen und Konflikten am Arbeitsplatz führt, "weil die Leute sehr gestreßt zur Arbeit kommen"28. Einige Mitarbeiter beschreiben, daß Probleme des häuslichen Alltags in die betriebliche Sphäre hineingetragen werden und von den eigentlichen Arbeitsaufgaben ablenken. Die Abteilungsleiterin der Materialbeschaffung erklärt, daß man vor allem darüber spricht, "daß kein Geld vorhanden ist und daß man gezwungen ist, jeden Tag daran zu denken, womit kann ich heute meine Kinder füttern? Die Gedanken drehen sich ums Essen." Ein Mitarbeiter aus der Abteilung Konstruktion/Arbeitsvorbereitung beschreibt, wie aus der persönlichen Notlage vieler Mitarbeiter Rivalitäten untereinander entstehen:

(Dolmetscherin:) Die personelle Spannung ist angewachsen, Konflikte haben zugenommen und zwar aus allen Gründen. Nicht nur aus solchen Gründen, daß es kein Geld gibt und diese Konflikte aus der beruflichen Sphäre kommen, sondern daß Leute einfach sich gegenseitig beneiden oder auch hassen oder ihre Lasten nicht mit sich herumtragen können. Daß sie zu Hause kein Wasser haben.

(Interviewerin:) Das war früher nicht so?

(Dolmetscherin:) Das war früher nicht so. Ehm, wenigstens hatten die früher zu Hause, naja alles paletti, zu Hause war alles paletti, und deswegen haben sie sich bei der Arbeit keine Sorgen gemacht. Er meinte noch zu mir: Früher, bei der Arbeit, hatte er nur an die Arbeit gedacht, jetzt denkt er nur daran, wo kann ich jetzt mir Geld beschaffen? Und deswegen werden die Leute nervös, weil sie nur daran denken und beschreien sich gegenseitig. Also, sie können sich nicht zusammenreißen.

(Interviewerin:) Weil jeder so mit eigenen Problemen beschäftigt ist?

(Dolmetscherin:) Genau, genau. Jeder plagt sich mit seinen eigenen Problemen hier rum, und deswegen können sie sich gegenseitig nicht verstehen, weil sie etwas ganz anderes denken.

(Interviewerin:) Es wäre doch aber auch denkbar, daß alle dieselben Probleme haben und daß sie deswegen zusammenhalten.? Das ist aber nicht so?

(Dolmetscherin:) Das tun sie aber nicht. Die sind egoistischer geworden. Sein Eindruck.

(Interviewerin:) Obwohl sie nichts haben?

(Dolmetscherin:) Genau. Was die egoistischer macht, ist die Möglichkeit, an der Seite Geld zu beschaffen. Entweder ich oder du.

(Interviewerin:) Aha, also nicht innerhalb des Betriebes, sondern außerhalb?

28 Abteilungsleiter der Produktionsabteilung 
(Dolmetscherin:) Nicht innerhalb. Nur außerhalb des Betriebes.

Zum anderen führt die stagnierende Produktion zwar einerseits dazu, daß der in den 70er und 80er Jahren herrschende Leistungsdruck reduziert wurde, doch entstehen gleichzeitig Langeweile und Unzufriedenheit: "Einerseits ist es besser geworden, weil die Spannung etwas nachgelassen hat, und zum zweiten ist es schlimmer geworden, weil die Leute, na ja, gar nicht wissen, wofür sie jetzt arbeiten müssen." ${ }^{29}$ Die oben erwähnten Konflikte breiten sich auch aufgrund mangelnder Beschäftigungsmöglichkeiten weiter aus. Ein Mitarbeiter aus der Abteilung Konstruktion/Arbeitsvorbereitung schildert diesen Zusammenhang wie folgt:

(Dolmetscherin:) "Man hat gar nichts zu diskutieren, und man hat dann keine Konflikte zu sehen. Allerdings arbeiten hier sehr viele Frauen, und wenn eine schief geguckt hat oder was Besseres anhat, dann wird nur deswegen lange rumdiskutiert.

(Interviewerin:) Das ist jetzt so. Weil es keinen anderen Stoff gibt. Also, wenn jemand sich unterscheidet, dann ist das Konfliktstoff?

(Dolmetscherin:) Genau. Also unterscheidet in welcher Hinsicht. Sie ist besser angezogen, und sie hat mich beschimpft gestern, und dann beschimpfe ich sie heute. Und werde dann ein paar Freundinnen heranziehen. Dann können wir schön lästern, also dann haben wir wenigstens etwas, womit wir uns den Tag vertreiben können. Er versteht es so, daß die Leute nichts persönliches gegeneinander haben, bloß versuchen, irgendwie die Zeit zu vertreiben. Sie sind dann nicht kreativ irgendwie, daß sie ihre Zeit für etwas besseres nutzen, weil es nämlich Frauen sind, die auch kein Interesse an ihrer Arbeit haben, dann labern sie den ganzen Tag. Ja, und der starke Druck von früher fehlt. (...) Allerdings heutzutage spielen Familiensorgen eine ganz große Rolle und lenken ab von der Arbeit, von der Routinearbeit."

Führungskräfte sind heute mehr denn je einem tiefen Mißtrauen von seiten der Mitarbeiter ausgesetzt, was teilweise dadurch erklärbar ist, daß Führungskräfte privilegierten Zugang zu lukrativen zwielichtigen Initiativen haben. Ein Mitarbeiter aus der Abteilung Arbeitsvorbereitung/ Konstruktion beschreibt das Verhältnis zu Führungskräften folgendermaßen:

(Dolmetscherin:) "Er meinte, daß in jedem Privatgespräch heutzutage die Führungskräfte und der Generaldirektor verpönt werden. Man stellt sich die Frage, woher hat er denn das Geld, die Leute im Unternehmen haben das Geld nicht, und er baut sich ein großes Haus. Und das gleiche kann man auch auf seine Stellvertretung beziehen. Also, es wird aber nur so rumerzählt, er kann nichts beweisen. Und außerdem stört es sehr stark, daß die Vorgesetzten

${ }^{29}$ Mitarbeiter der Abteilung Konstruktion/Arbeitsvorbereitung 
gewisse Lieblinge haben, und die Lieblinge werden bevorzugt. Die bekommen als erste Geld, die bekommen bessere Stellungen...

(Interviewerin:) War das früher auch so?

(Dolmetscherin:) Früher es gab keine offenen Auftritte seitens der einfachen Mitarbeiter. Falls es doch irgendwelche gab, dann war diese Kritik zu sanft. Heutzutage kann man schon offen reden, aber es wird sowieso nichts gemacht, ob man seine eigene Meinung geäußert hat oder nicht.

Kann man denn behaupten, daß die Geschäftsleitung meinte, es sitzen alle in einem Boot? Nein, wir sitzen in zwei verschiedenen Booten. Es gibt eine ganz große Distanz zwischen dem Generaldirektor und den einfachen Mitarbeitern. Früher war es besser in dieser Hinsicht, weil der Generaldirektor jedem persönlich zu seinem Jubliäum gratuliert hat oder einfach zu den Arbeitern runterkam und die Probleme formal besprochen hat.

(Interviewerin:) Also gab es früher nur ein Boot und heute zwei?

(Dolmetscherin:) Genau. Früher gab es ein Gefühl der Zusammengehörigkeit, heutzutage erschien er nur einmal und hat jemand zu seinem 50. Geburtstag gratuliert. Ansonsten sieht man den kaum, und es wird auch mit dieser Art und Weise, nicht zu erscheinen, suggeriert, daß jeder seinen eigenen Weg hat.

(Interviewerin:) Ähm, wenn es früher nur ein Boot gab und heute zwei, wer sitzt denn in dem zweiten drin? Nur der Generaldirektor alleine oder der Generaldirektor und alle Führungskräfte?

(Dolmetscherin:) Der Generaldirektor und seine ersten Führungskräfte, also seine Stellvertretung...

(Interviewerin:) Ach so.

(Dolmetscherin:) ...und im zweiten Boot Mitarbeiter und Abteilungsleiter."

Der Führungsstil insgesamt wird von den meisten Mitarbeitern, aber auch von Abteilungsleitern, als unverändert autoritär wahrgenommen, wie z. B. die Äußerung der Abteilungsleiterin Materialbeschaffung zeigt:

"So wie sie früher nicht frei sprechen konnte, genauso kann sie, genauso schlecht kann sie heute frei sprechen. Ansonsten werden irgendwelche Sanktionen seitens der Verwaltung oder des Vorgesetzten erfolgen."

\subsection{Was tun? Ansatzpunkte für Führungskräfte}

Betrachtet man die berichteten Handlungsmuster unter der Perspektive einer möglichen Bewältigung der bedrohlichen Situation, so liefert die skizzierte Bewußtseinslage ein recht düsteres Bild. Die Option des Abwartens ist wenig geeignet, die anstehenden Probleme zu lösen; vielmehr wirkt Handlungsverzicht in der gegenwärtigen Lage zumindest problemstabilisierend (Pfeil 1 in Abb. 2). 
Speziell die vorherrschende Variante des Handlungsverzichts, die mit Hoffnung verbunden ist, dürfte sich darüber hinaus tendenziell selbst verstärken: Je schwieriger die Lage erscheint, desto mehr verstärkt sich kompensierend die Hoffnung auf eine "Erlösung" durch "höhere Mächte".

Die Alternative "Flucht" wirkt in ihrer Extremform, wenn etwa Mitarbeiter das Unternehmen zugunsten lukrativer Geschäfte im "Handel" verlassen oder gar das Unternehmen durch Schwarzmarktgeschäfte schädigen, insofern problemeskalierend, als die letzten vorhandenen Ressourcen systematisch vernichtet werden (Pfeil 2). Wie das Engagement des Tochterunternehmens von Malachowka zeigt, kann eine abgeschwächte Form dieser Flucht, kombiniert mit positiver Initiative ("Angriff"), jedoch durchaus als ein Schritt gewertet werden, die Probleme des Unternehmens zu entschärfen. Die einzige Handlungsstrategie, die uneingeschränkt problemlösend bzw. problementschärfend wirken könnte, ist das beschriebene Angriffsverhalten (z. B. Personalumschulung). Bezogen auf die Abb. 2 führt diese positive Initiative dazu, daß sich die Situation de facto entspannt (Pfeil 3) und damit auch die erste Bewertung als weniger bedrohlich ausfällt. Im Sinne eines Versuchs zur Verbesserung der Situation müßte man also die Frage stellen, was Führungskräfte tun können, um das Reaktionsmuster "Angriff" zu stärken.

In diesem Zusammenhang ist die Frage von Bedeutung, welche Qualität den erfragten Bewußseinslagen beigemessen wird. Die geschilderten Einschätzungen spiegeln vermutlich einerseits objektive Realitäten wider; andererseits können ihnen aber partiell irrtümliche subjektive Einschätzungen der Befragten zugrunde liegen, die unabhängig von den objektiven Gegebenheiten sind. Möchte man im Ergebnis die Option "positive Initiative" verstärken, so kommt es also darauf an, die Frage zu klären, welche Gründe der weitgehenden Ablehnung dieser Option von seiten der Befragten zugrundeliegen. Nachfolgend werden drei Alternativen erörtert: primär objektive Gründe, die nicht vom Unternehmen zu beeinflussen sind; primär objektive Gründe, die vom Unternehmen beeinflußt werden können; primär subjektive Gründe, die sich auf psychologische Prozesse zurückführen lassen und die ebenfalls beeinflußbar sind. Je nach Art der Begründung ergeben sich entsprechend unterschiedliche Folgerungen.

Die aussichtsloseste Variante wäre, daß die wahrgenommene geringe Situationskontrolle tatsächlich Widerspiegelung objektiver Grenzen der Verbesserung der Situation ist und insofern die subjektive Erklärung der Befragten mit den objektiven Gegebenheiten übereinstimmt. In welchem Maß dies zutrifft, ist nicht mit Sicherheit zu beantworten, doch gibt es auch in der Literatur (vgl. z. B. Peng/ Heath 1996) ausreichend Hinweise darauf, daß zumindest teilweise auch objektiv eine Verbesserung der Lage an Grenzen stößt. Exemplarisch seien drei Grenzen beschrieben, die speziell für Malachowka gelten. Angesichts zusammengebrochener Absatzmärkte für 
Bergbauprodukte scheint die mehrfach geplante Umrüstung auf Konsumgüter eine aussichtsreiche Strategie, doch fehlen einerseits Beschaffungsmöglichkeiten für entsprechende Rohstoffe und Energien, andererseits fehlt das Geld für die notwendigen Investitionen in Maschinen und Personal, weil in Rußland (noch) kein entsprechender Kapitalmarkt existiert. Drittens werden (legale) unternehmerische Erfolge insofern systembedingt bestraft, als Gewinne durch willkürliche Steuerpolitik und mafiöse Einflüsse im Wirtschaftssystem systematisch geschmälert bzw. vernichtet werden. Alle beschriebenen Grenzen liegen in den Rahmenbedingungen außerhalb des Unternehmens und sind durch unternehmerische Initiative allein kaum zu beheben. Legt man diese Variante zugrunde, so sind Ratschläge zur Förderung positiver Initiative in der Tat problematisch, da nahezu aussichtslos.

Eine zweite Variante ist denkbar, bei der die wahrgenommen Grenzen aus Sicht des Unternehmens beeinflußt werden können. Dies würde bedeuten, daß die Möglichkeiten zur Behebung der Mißstände (z. B. durch Finanzmanagement, Marketing, Qualitätsmanagement, Kooperation mit westlichen Unternehmen etc.) entweder nicht hinreichend bekannt sind oder zwar bekannt sind, aber mangels Erfahrung nur unzureichend umgesetzt werden. In diesem Fall bestünde vor allem ein Aufklärungs- und Qualifizierungsproblem hinsichtlich betriebswirtschaftlicher Methoden eines angemessenen Krisenmanagements. Führungsbezogen müßten zusätzlich Überlegungen angestellt werden, wie die Situationskontrolle der Mitarbeiter objektiv angehoben werden kann. In diesem Zusammenhang sind auch Führungsschulungen wichtig: Es geht darum, den Mitarbeitern den Mut zur kritischen Loyalität zu vermitteln, um das im Unternehmen vorhandene Innovationspotential zu aktivieren. Vor dem Hintergrund des erwähnten Mißtrauens gegenüber Kollegen und gegenüber Führungskräften scheinen die Voraussetzungen hierfür allerdings ungünstig, weil eine innovationsfördernde kritische Loyalität in hohem Maße Vertrauen erfordert. Insofern ergibt sich im Ergebnis eine eklatante Schere zwischen dem Innovationsbedarf einerseits und den psychologischen Voraussetzungen zur Förderung des hierzu erforderlichen kritischen Dialogs andererseits.

Drittens besteht die Möglichkeit, daß die Blockierung von Handlungsmöglichkeiten nur eine subjektive Wahrnehmungsverzerrung der Befragten reflektiert (und nicht eine objektive Handlungsbegrenzung). In diesem Fall glauben die Handelnden irrtümlich, daß sie wenig Situationskontrolle besitzen, und verzichten angesichts dieser Grundannahme darauf, die Möglichkeiten von "Angriff" und "Flucht" auch nur zu durchdenken, obwohl Handlungsmöglichkeiten bestehen. Im Zusammenhang mit der berichteten Erfahrung von wenig Situationskontrolle in der Vergangenheit (vgl. oben) könnte dieses Muster in Rußland ein durch Erfahrung verstärktes CopingVerhalten sein, das zudem deshalb attraktiv ist, weil es die abwartende Haltung der Befragten subjektiv legitimiert und stabilisiert. Was von den Befragten als 
"russische Mentalität" beschrieben wird, erscheint uns daher weniger als eine unveränderliche Charaktereigenschaft, sondern vielmehr als eine Einstellung, die teilweise durch Erfahrung erlernt wurde und deshalb im Prinzip veränderbar ist. Nachdrücklich sei an dieser Stelle noch einmal erwähnt, daß der häufige Rekurs der Interviewten auf die russische Mentalität eine Zuschreibung darstellt, die nicht den objektiven Gegebenheiten entsprechen muß. Der Tatbestand aber, daß derartige Zuschreibungen vorgenommen werden, ist ein wichtiges Untersuchungsresultat an sich, da diese Zuschreibung auf seiten der Interviewten handlungsleitend wirken. Deshalb müßte in diesem Fall den Befragten die korrigierende Erfahrung vermittelt werden, daß diese Zuschreibung und die These der Handlungsblockierung relativiert werden müssen. Um dies zu erreichen, dürfte allein die Vermittlung kognitiven Wissens nicht ausreichen; es müßte zusätzlich dafür Sorge getragen werden, daß die Betroffenen dieses Wissen als vor Ort erfolgreich umsetzbar erleben.

Für das Ausbleiben der positiven Initiative sind vermutlich alle drei Erklärungen verantwortlich. Während die Rahmenbedingungen des Systems eine objektive Grenze darstellen, die von seiten des Unternehmens nicht zu beeinflussen und darüber hinaus schwer kalkulierbar ist, setzen die meisten westlichen Ansätze in Form von Managementtrainings an der Aufklärungs- und Qualifizierungsvariante an. Die Perspektive der dritten Variante, die auch kulturspezifische grundlegende Einstellungen zur Erklärung mit heranzieht, wird dagegen bisher wenig in Betracht gezogen.

\section{Literatur}

Bayer, J. (1991): Vom latenten Pluralismus zur Demokratie, in: Deppe, R./ Dubiel, H./ Rödel, U. (Hrsg.): Demokratischer Umbruch in Osteuropa. Frankfurt/ Main: Suhrkamp, S. 151-166.

Boerner, S./ Gebert, D./ Agafonov, J. (1996): Rußland auf dem Weg in die offene Gesellschaft? - Interviewprotokolle einer Untersuchung in einem Moskauer Unternehmen im Herbst 1996. Unveröffentlichtes Manuskript, TU Berlin.

Bogacheva, N: (1993): The russian association of business schools (RABS), in: efmd Forum 93/2, S. 8.

Cassel, D. (1988): Wirtschaftspolitik als Ordnungspolitik, in: Cassel, D. (Hrsg.): Ordnungspolitik, München: Vahlen.

Chadwick, J. (1993): Sundridge Park and the Russians - Learning from each other, in: efmd Forum 93/2, S. 43-45.

Dittrich, E. J. (1992): Arbeitsbeziehungen in Osteuropa: Vorschläge zu ihrer Analyse in: Dittrich, E. J./ Haferkemper, M./ Schmidt, G./ Stojanov, Ch. (Hrsg.): Der Wandel industrieller Beziehungen in Osteuropa. New York: Campus, S. 91-106.

Gebert, D./ Boerner, S. (1995): Manager im Dilemma - Abschied von der offenen Gesellschaft? Frankfurt am Main: Campus. 
Götz, R. (1991): Auf dem Weg zum autonomen Unternehmen? Wirtschaftsrechtliche und betriebswirtschaftliche Aspekte der sowjetischen Wirtschaftsreform. Berichte des Bundesinstituts für ostwissenschaftliche und internationale Studien. Herausgegeben vom Bundesinstitut für ostwissenschaftliche und internationale Studien, Köln.

Haferkemper, M. (1992): Voraussetzungen für den Wandel industrieller Beziehungen - Überlegungen zu osteuropäischen Variationen eines "westlichen" Forschungsthemas, in: Dittrich, E.J./ Haferkemper, M./ Schmidt, G./ Stojanov, Ch. (Hrsg.): Der Wandel industrieller Beziehungen in Osteuropa. New York: Campus, S. 143-166.

Hensel, K. P. (1992). Grundformen der Wirtschaftsordnung. Marktwirtschaft Zentralverwaltungswirtschaft. 4. Aufl. München: Vahlen.

Hentze, J. / Lindert, K. (1992): Manager im Vergleich. Daten aus Deutschland und Osteuropa. Bern: Haupt.

Lazarus, R. S. (1991): Emotion and adaptation. New York: Oxford University Press.

Manoukovsky, A. (1993): Russian management: How far from the West? in: efmd Forum 93/2, S. 28-33

Nielsen, F.J. (1992): Soviet Union, in: Ökonomisk Institut, Aartus Universität, S.112-156.

Peng, M.W./ Heath, P.S. (1996): The growth of the firm in planned economics in transition: institutions, organizations, and strategic choice, in: Academy of Management Review, Vol. 21, No. 2, S. 492-528.

Pieper, E. (1993): Managementtraining in Osteuropa. Wiesbaden: Gabler.

Puffer, S. M. (1994): Understandig the bear: a portrait of Russian business leaders, in: Academy of Management Executive, Vol. 8, No.1, 41-54.

Shubkin, V. (1992): Arbeitsbeziehungen in der UdSSR: Vom nicht-ökonomischen Zwang zur ökonomischen Stimulierung, in: Dittrich, E.J./ Haferkemper, M./ Schmidt, G./ Stojanov, Ch. (Hrsg.): Der Wandel industrieller Beziehungen in Osteuropa. New York: Campus, S. 73-88.

Staniszkis, J. (1991): Dilemmata der Demokratie in Osteuropa, in: Deppe, R./ Dubiel, H./ Rödel, U. (Hrsg.): Demokratischer Umbruch in Osteuropa. Frankfurt/Main: Suhrkamp, S. 326-347.

Stojanov, Ch. (1992): Die postsozialistische Transformation - Eine eigenartige (Re-) Modernisierung? in: Dittrich, E.J./ Haferkemper, M./ Schmidt, G./ Stojanov, Ch. (Hrsg.): Der Wandel industrieller Beziehungen in Osteuropa. New York: Campus, S. 19-42.

Szeloch, Z. (1993): Aus- und Weiterbildung von Managern in Russland, Weissrussland und der Ukraine, in: Mahari, J. I./ Schade, M. (Hrsg.): Das Jahrbuch der ManagementWeiterbildung. Seminare 1993, 4. Aufl. Hamburg, S. 196-206.

Tatur, M. (1992): Industrielle Beziehungen und sozialer Konflikt in sozialistischen Gesellschaften - Ein Vergleich Polens und der Sowjetunion, in: Dittrich, E. J./ Haferkemper, M./ Schmidt, G./ Stojanov, Ch. (Hrsg.): Der Wandel industrieller Beziehungen in Osteuropa. New York: Campus, S. 189-212.

Vlachoutsicos, Ch. A./ Lawrence, P. R. (1996): How managerial learning can assist economic transformation in Russia, in: Organization Studies, 17/2, S. 311-325. 
Welsh, D. H./ Luthans, F./ Sommer, S. M. (1993): Managing russian factory workers: The impact of U. S.-based behavioral and participative techniques, in: Academy of Management Journal, Vol. 36, No. 1, S. 58-79. 